\title{
Vorwort und Einleitung.
}

Die Darstellung und Benutzung der Metalle bei den sogenannten Naturvölkern ist noch nicht im Zusammenhange und mit Rücksicht auf den Vergleich behandelt worden. Und doch bietet dieses Thema nicht allein vom ethnographischen und allgemein kulturhistorischen Standpunkte ans ein hohes Interesse; auch bei der Beurteilung prähistorischer Fragen ist es von Wichtigkeit zu wissen, wie die prinitiven Völker zur Kenntnis der Metalle gelangen, wie sie dieselben erschmelyen und benutzen, denn hier eröffnet sich die Aussicht, auf dem Wege der Analogie wertrolle Ergebnisse zu gewiunen.

Wie bei so vielen ethnographischen Dingen, ist es auch auf diesem Gebiete die höchste Zeit, zu sammelı und zu retten, was noch vorhanden ist. Europäische und amerikanische Metalle dringen bei erleichtertem Verkehr bis in die fernsten Errdenwinkel und vernichten altheimische Industrien der Naturvölker. Schon erlegt der centralafrikanische Schwarze den Elefanten mit dem Hinterlader und die weltberühmten Damaszenerklingen von Schiras und Meschhed in Persien werden nur noch aus russischem Eisen geschmiedet. Die einheimische Metallindustrie der meisten Naturvölker ist auf den Aussterbestand gesetzt, sie ist den billigeren und besseren europäischen Erzeugnissen gegenüber nicht mehr konkurrenzfähig, die letzte Stunde naht für sie und noch, so fürchten wir, ist manche wichtige Thatsache nicht eingeheimst, die uns AufschluB zu geben vermöchte über das ursprüngliche Verfahren in diesem oder jenem Zweige der Metalltechnik. Von den Reisenden, auf deren Berichte wir zum großen Teile angewiesen sind, ist im allgemeinen 
nur wenig Autmerksamkeit dem uns hier interessierenden Gegenstande zugewendet worden, einmal, weil die hüttenmännische Einsicht den meisten mangelt und dann, weil die Wichtigkeit der Sache für prähistorische Fragen erst neuerdings erkannt wurde, zunal seit Christian Hos'rmann in seiner vernichtenden Kritik der Inreiperiodenteilung mit Krfolg auf die Bedeutung der Metallurgie bei den Naturvölkern hinwies. Wenige Ausnahmen abgerechnet, unter denen einer der genialsten Reisenden der Gegenwart, Georg Schweinfurtr, hervorragt, sind wir meist auf dürftige Berichte angewiesen, die uns das Bild der I)arstellung und Benutzung der Metalle bei den Naturvölkern liefern müssen. Wünschenswerte krgänzungen bringen die in unseren Museen aufgestapelten Schätze.

Sehr wohl ist der Verfasser sich bewußt gewesen, daß bei der Behandlung der so weitschichtigen und in die verschiedensten Wissensgebiete eingreifenden Aufgabe eigentlich nur mit vereinten Kräften etwas vollständiges zu erreichen ist und daß ein einzelner hier nicht zum Abschluß gelangen kamn. Geognosie und Geographie, Ethnographie, Hüttenkunde, Chemie, Prähistorie und Linguistik -- alle diese Wissenschaften verlangen Berücksichtigung bei der Bearbeitung unseres 'Themas, und wo wäre der Manm, der von sich sagen dürfte, er beherrsche sie gleichmäBig? Da wird jeder nach seinem Wissensstandpunkte auf Lücken stoßen. Aber doch mußte der Anfang gemacht und das Gebäude wenigstens aus dem Rohen heraus gestaltet werden. So gebe ich denn, was ich fand, als. Beiträge, Stoff und Grundlage für den weiteren Ausbau.

Der europäische und der semitische Kulturkreis sind in der vorliegenden Arbeit ausgeschlossen. Was die Metalle innerhalb derselben betrifft, so haben so zahlreiche Gelehrte sich damit beschäftigt und die interessierenden Fragen der Lösung nahe gebracht, daß auch nicht einmal von einer Rekapitulation die Rede sein konnte; auch wird sich im Verlaufe der Darstellung zeigen, daß die Einwirkung jener wichtigsten Kulturkreise unserer Erde auf die Metallindustrie der Naturvölker eine kaum nennenswerte war, ja, daß die letzteren, bis auf die neue, umgestaltende Zeit, fast ganz unberührt von jenen blieben. Dagegen war es des Vergleiches wegen geboten, die ostasiatischen und amerikanischen Kulturvölker in die 
Betrachtung einzubeziehen und zu fragen, ob sie von Einfluß auf die Metallurgie benachbarter Naturvölker waren: aber auch jene zeigen in bezug auf die Metalle abgeschlossene Reiche mit geringen oder gar keinen Ausstrahlungen auf die Nachbarn.

Die Metalle, welche wesentlich ins Auge zu fassen waren, sind Eisen, Kupfer, Zinn und die Legierung aus den beiden letzteren, die Bronze. Um diese drehen sich wichtige wissenschaftliche Streitfragen, sie sind es, die in kultureller Beziehung vor allen anderen in Betracht kommen, während die edlen Metalle eine geringere Rolle spielen, auch bei ihnen sich noch kein Streit um „Entlehnung der Kenntnis" erhoben hat, ihr Vorkommen im augenfälligen gediegenen Zustande einen solchen auch unnötig machte.

Geographisch vorschreitend, beginne ich den Rundgang mit den alten Ägyptern, denen neben der Bronze in den ältesten Zeiten zweifellos das Eisen bekannt war. Daß von ihnen die Eisenkenntnis zu den benachbarten Nigritiern gelangte, läßt sich keineswegs mit Bestimmtheit behaupten, eher neige ich der Ansicht zu, daB die Eisenbearbeitung ein durchaus ursprüngliches Gewerbe der Neger ist, die ein „Eisenreich“ für sich bilden, von so ausgeprägter Entwickelung, daß neuerdings ein durch wenig Kritik ausgezeichneter Kopf alle Eisenindustrie von den Schwarzen abzuleiten versucht. ${ }^{1}$ In Afrika folgte das Eisen direkt auf den Stein und zwar entwickelte sich die Eisendarstellung im Nordosten oder in Centralafrika, von wo sie erst spät nach dem Süden gelangte. Kupfer, wiewohl es auch von den Negern erschmolzen wird, ist nur auf wenige Gebiete beschränkt, von denen aus es auf dem Handelswege verbreitet wird. Es ist höchstens gleichalterig mit dem Eisen bei den Nigritiern, und von einer dem Eisen vorangehenden „Kupferperiode“, geschweige denn von einer „Bronzeperiode“ kann in Afrika keine Rede sein.

Vorderindien bietet ein abgeschlossenes Reich für sich. Auch hier ist eine Steinzeit nachweisbar und eine Einführung der Metalle

1 Le fer, comme emploi industriel, est originaire d'Afrique. En effet, e'est en Afrique seulement (!!) que nous rencontrons des peuples saurages, connaissant l'emploi du fer, sachant le produire et travailler. Dieser Satz des Herrn Gabriel de Mortillet (Bulletins de la soc. d'Anthropol. 1883. 562) zeigt wiederum die große Oberflächlichkeit des bei uns noch ernst genommenen Mannes. 
von außen her nicht zu erkennen. $\mathrm{Da} B$ Vorderindien das Stammland aller Bronze gewesen sein soll (WoRsAAE), erweist sich als eine willkürliche Annahme. Alte Bronzen gehören dort zu den größten Seltenheiten; sie sind von ganz anderer Zusammensetzung als unsere Bronzen und kommen zusammen mit Eisen vor. Vorderindien war in alter Zeit kein "Bronzeland“, es bezog selbst im Altertum sein Zinn aus dem fernen Abendlande, denn die reichen und näher liegenden hinterindischen Zinnvorkommnisse waren damals wohl noch kaum erschlossen. Dagegen deuten häufige alte Kupferfunde auf das hohe Alter dieses Metalles in Indien, das heute dort, ebenso wie das Eisen, noch nach uralter Art erschmolzen wird nach Methoden, die in mancher Beziehung an jene der Nigritier erinnern, ohne daß dabei an Entlehnung gedacht zu werden braucht. Ob Eisen, ob Kupfer das ältere Metall in Vorderindien war - wer vermag das heute mit Sicherheit zu entscheiden? Zwar spricht sich die vergleichende Sprachforschung zu Gunsten des Kupfers aus, aber die Sicherheit ihrer Entscheidung läßt manches zu wünschen übrig. Als ein AusfluB der indischen Metallarbeit ragen in unser europäisches Kulturleben die konservativen Zigeunerschmiede hinein mit uralten Methoden und Instrumenten; ihnen ist eine besondere Betrachtung gewidmet, welche allerdings von des sonst verdienten BataillaRd's Phantasien, daß nämlich die Zigeuner die Verbreiter der alten Bronzekultur in Europa waren, nichts wissen mag.

Abermals ein selbständiges metallurgisches Reich bilden die malayischen Völker. Ihr wohlcharakterisiertes, seit uralter Zeit bei ihnen heimisches Verfahren der Eisenbereitung reicht von Madagaskar bis Nenguinea und im Norden bis zu den Philippinen. Eisen ist ihr ältestes Metall. Kupfer, das sie gleichfalls, aber weniger darstellen, erscheint später und ebenso die Bronze.

Hinterindien, von wo die uns angehenden Nachrichten spärlich Hießen und wo das Studium der Metalle bei den hochinteressanten Aboriginern des Innern eine dankbare Aufgabe bilden würde, tritt uns mit prähistorischen Zeugen der jüngeren Steinzeit in Gesellschaft von Bronzen entgegen und deutet durch die Verschiedenartigkeit der Methoden, nach denen seine Urvölker (in Kambodja und Birma) das Eisen gewinnen, auf eine selbständige und ursprüngliche Darstellnng desselben, ohne erkennbare fremde Einflüsse. 
Für das in seiner Kultur völlig isoliert dastehende China wird bereits vor 3500 Jahren eine hochentwickelte Bronzeindustrie bezeugt und Sinologen sind geneigt, der Bronze dort die Priorität vor dem Eisen zuzuerkennen - ob aber nicht unter dem Einflusse skandinavischer Anschauungen? Eisen ist in der älteren chinesischen Litteratur neben Zinn und Kupfer gleichfalls ein durchaus bekanntes Metall und die chinesische Eisendarstellung erscheint uns noch jetzt als eine ganz eigentümliche, von der aller übrigen Völker völlig geschiedene und selbständige. $\mathrm{Da} \beta$ aber die Chinesen, die in so vielen Dingen die Lehrmeister der Japaner gewesen, letzteren auch die Eisenkenntnis übermittelt haben sollten, läßt sich kaum annehmen: denn Japan zeigt in dieser Richtung ein ganz anderes Verfahren als China, nämlich die Eisenschmelzung in Öfen, während China bis zum heutigen Tage nur in kleinen Schmelztiegeln sein Eisen gewinnt. Für China sind die prähistorischen Verhältnisse noch wenig oder gar nicht studiert, wiewohl wir wissen, daß auch dieses Land seine Steinzeit hatte; in Japan aber, wo Europäer einflußreich wirken und Gelegenheit zu Studien haben, erkannte man die große Ähnlichkeit der dortigen vorgeschichtlichen Funde mit jenen Europas, die Übereinstimmung der zugehauenen und polierten Steingeräte, gesellt mit Bronzen, welche letztere man auch in Japan für älter als das Eisen anspricht.

Licht beginnt sich zu verbreiten über den Norden Asiens in prähistorischer Zeit. Nicht alle sibirischen Völkerschaften befanden sich, als die russischen Entdecker in das Land kamen, im Zustande der Steinzeit; einzelne Stämme verstanden es bereits, das Eisen zu reduzieren und $\mathrm{zu}$ schmieden, wohl als ein Erbteil türkischer Völker, die, aus Centralasien kommend und als Eroberer eindringend, die Eisenkunde mitbrachten. Aber lange vor den eisenkundigen Türkvölkeru hatten vom Ural bis zum Altai finnische Stämme, die in der Tradition als „Tschuden“ fortleben, eifrig Bergbau und Metallschmelzerei betrieben. Kupfer war ihr Hauptmetall, das sie zu schmelzen und gießen verstanden. Neben dem Kupfer der Tschuden und dem Eisen der Türken erbielt sich aber im fernen Osten der alten Welt, da, wo diese sich Amerika nähert, die Steinzeit, welche erst den erobernden Russen wich und bei den Tschuktschen in ihren letzten Ausläufern heute vor unseren Augen dahinsiecht. 
Nicht geleugnet kann werden die Finheit des Menschen in der alten und neuen Welt. Aber die Differenzierung beider liegt so weit zurück, daß von einer gemeinsamen Quelle ihrer beiderseitigen Metallkenntnisse keine Rede sein kann. Oder, wemn man grundlos diese Kenntnis von' der alten nach der neuen Welt gelangen ließ, warum dreht man, mit gleich gutem Grunde, die Sache nicht einmal um und läßt die Inkaperuaner die Bronzelehrmeister der Asiaten werden? Das gäbe doch Abwechselung. Auch in der nenen Welt zeigen sich die „Metallreiche“ unabhängig von einander. Eisen kanute man im vorkolumbischen Amerika nicht, wenigstens kein künstlich dargestelltes; aber Meteoreisen wurde wiederholt, so namentlich von den Eskimos, benutzt und auf ähnlicher Stufe stand auch die Anwendung des gediegenen Kupfers in Nordamerika. Es wird von den Indianern wie weicher Stein verarbeitet und kennzeichnet höchstens die Grenze zwischen Stein- und Metallzeit. Der große Kulturfortschritt, die Erze mit Kohlen zu reduzieren und die Metalle im Feuer zu behandeln, ist dreimal in Amerika gemacht worden: in Mexiko, in Cundinamarca und in Peru, stets aber selbstständig und unabhängig von einander. In Mexiko war Kupfer das Hauptmetall, seltener war Bronze und beide wurden noch neben dem Stein benutzt, im ganzen auch, wie die spärlichen Funde beweisen, nicht häufig. Weiter war man in bezug auf die Bronze in Peru, wo umgekehrt die Kupfergeräte seltener sind. Alle metallurgischen Arbeiten dieser amerikanischen Kulturvölker wurden ohne Gebläse ausgeführt. I)ie Analysen der Bronzen ergeben eine große Verschiedenheit in der Zusammensetzung und keinerlei Übereinstimmung zwischen mexikanischen und peruanischen Erzeugnissen.

In alle die hier aufgezählten Länder, den größeren Teil unserer Erde, brauchten die Europäer nicht erst die Metalle zu bringen, weil sie selbständig dort entdeckt und verarbeitet worden waren. Das Eisen freilich haben sie in Amerika eingeführt; der Nordwesten erhielt es ziemlich spät durch die Russen, in die übrigen Gebiete hatten sich Spanier, Portugiesen und Briten geteilt. Den Peruanern und Mexikanern war dasselbe nur „schwarzes Kupfer“. Metallos war die Südsee, deren zahlreiche Inselfluren sich über ein Gebiet von hundert Längengraden erstrecken und wo zunächst die Spanier mit der Verbreitung des Eisens begannen. Aber volle drei Jahr- 
hunderte hat hier der ProzeB der Metallverbreitung in Anspruch genommen, denn erst das achte Jahrzehnt unseres Säkulums sah den AbschluB auf Neuguinea, dessen Bewohner die letzten unseres Erdballes waren, welche in die Metallkenntnis eingeführt wurden.

Überblicken wir alle Gebiete, die wir mit Rücksicht auf die Metalle durchwandert haben, so vermögen wir wohl eine große Abwechselung, nirgends aber die „gesetzmäBige Reịhenfolge“ von Stein, Bronze, Kisen zu entdecken. Bei den Naturvölkern, die wir jetzt in ihrem Verhalten zur Metalldarstellung zu übersehen vermögen, ist kein Grund für die Anlegung einer solchen Zwangsjacke vorhanden. Die thatsächlichen Verhältnisse lassen da nichts Schablonenhaftes erkennen. Hat es doch schon an und für sich wenig Wahrscheinlichkeit, daß alle Völker in den verschiedensten Ländern und ganz unabhängig, ohne Verkehr mit einander, zu derselben Reihenfolge in der Erfindung der Metalle gelangt sein sollen: Kupfer, Zinn, Bronze, Eisen. Wir werden im Verlaufe der Darstellung sehen, daß gediegenes Kupfer, wo es vorhanden, von Naturvölkern im kalten Zustande zu Waffen und Geräten gehämmert wird; auch metallisches Zinn ist durch zufälliges Ausschmelzen bekannt geworden. Doch zur Mischung der beiden räumlich getrennten und nur durch den Verkehr zusammengeführten Metalle, zu ihrem kunstreichen Formen und Gießen gehört mehr, als im Durchschnitt bei Naturvölkern verlangt werden kann. Die Bronzedarstellung ist nicht so einfach und leicht, wie jene des Eisens, welches die primitivsten Völker zu erschmelzen wissen, während Bronze stets mit einem höheren Kulturgrad verknüpft ist. Daraus mag man sich die Parallele für unsere europäischen Vorfahren ziehen, die in ihrem primitiven Zustande sicher eher auf die Eisendarstellung, als auf das Komponieren und Formen der Bronze verfielen.

Eine zweite Lehre, die wir aus dem Verhalten der Naturvölker gegenüber den Metallen zu ziehen vermögen, betrifft die so beliebten Entlehnungstheorien. Wieviel Mühe und Gelehrsamkeit ist nicht aufgewendet worden, um die Metallkenntnis von einem Mittelpunkt gleichsam konzentrisch ausgehen, sie durch ein Volk zum anderen verbreiten zu lassen! Man braucht nur einmal die verschiedenen nach und nach aufgestellten „Ursprungsquellen“ und „Lehrmeister" zusammenzustellen und man wird da auf eine beträchtliche Anzahl 
Konkurrenten und auf die wunderlichsten Widersprüche stoßen. Ich glaube, daß auf die Entlehnung und das Übergehen der Metallkenntnis von einem Volke auf das andere noch zuviel Gewicht gelegt wird und daß dadurch weit schwierigere Verhältnisse künstlich geschaffen werden, als in der That vorliegen. Ohne für viele Fälle das Entlehnen und Lernen auszuschließen - sie liegen zu häufig offenkundig \%u Tage .., meine ich doch, daß uns ein gesunder Polygenismus weiter bringt, der die Metalle auch da erfunden sein läßt, wo sie in selbständiger Weise uns entgegentreten.

Leipzig, im Februar 1884.

Dr. R. Andree. 